\title{
Contrast as bearer of implicit meaning
}

\author{
Lioudmila Savinitch
}

Institute for information transmission problems RAS, Russia

https://doi.org/10.36505/ExLing-2018/09/0023/000356

\begin{abstract}
This paper analyzes contrast, modifier of communicative meaning, its accent structure, intonation contour, sound intensity, and use for conveying implicit meanings. We argue that contrastive highlighting of one of the utterances components in the given example is made by the speaker strategically, in order to convey occasional implicit meaning. All examples are illustrated with graphs displaying tone fluctuations and sound intensity.

Key words: prosody, intonation construction, contrast, implication
\end{abstract}

\section{Introduction}

While examining judicial discourse we focused on the speaker's different prosodic intention in two identical components of the utterance: first without contrastive highlighting and then with contrast. The question arose: was the accentual highlighting made accidentally or was it strategically realized, that is in order to express a definite communicative intention of the speaker? If strategically realized, then with what aim? Thus, the objectives of the present investigation were as follows: in the examples analyzed to identify the speaker's communicative strategies, semantics of the accents, prosodic characteristics of the accent bearers' word forms, and accent structure of the sentence.

Before proceeding to the analysis of our example, we shall give the definition of communicative strategy in Speech Acts theory:

The communicative strategy of a speaker consists of the choice of communicative intentions, the distribution of quanta of information on communicative components, and of the choice of the order of communicative components in a sentence" (Yanko 2001: 38).

The communicative strategies of a speaker are implemented in the structures of bearers of communicative meanings and can express intentions to make a statement, ask a question, make a request, give an order, etc. (Austin 1962; Searle 1976: 1-23).

Modifying communicative meanings are those meanings which do not belong to any category of basic illocutionary meanings, such as a statement, a question, a request, or an entreaty, but only modify the main types of illocution and their communicative components. These modifiers include contrast, verifying, or yes/no meaning, and emphasis.

ExLing 2018: Proceedings of $9^{\text {th }}$ Tutorial and Research Workshop on Experimental Linguistics, 28-30 August, Paris, Frannce 


\section{Characteristics of accented word forms}

Our example relates to the field of law and was recorded on audio media during a speech by a state prosecutor in court:

(1) Toktosunov \ Islambek $\boldsymbol{\nearrow}$ is accused of applying violence, not dangerous to life and health $\nearrow$, against a government representative $\ \boldsymbol{\nearrow}$ in the fulfillment of his official duties $\searrow$.

In this example the word forms highlighted in bold are accented bearers of communicative meanings; changes in fundamental frequency of the speech are indicated by arrows, which are located after the accented word forms. We will only select all accent bearers in the analyzed example and will specify some of their prosodic characteristics. Explicating the idea of 'intonation pattern' the present article proposes to introduce a concept of 'intonation construction' (Bryzgunova 1980: 96-122) that includes specific aspects of prosody, which will be considered in what follows.

At the beginning of the sentence two components of the proper name Toktosunov $\searrow$ Islambek $\nearrow$ serve as bearers of accents. The first accent bearer of this group is the word form Toktosunov $\downarrow$ with descending intonation of the 2nd Intonation Construction type (IC-2). This is characterized by a more intensive falling of pitch on a stressed syllable than in IC-1 type and a rising pitch on pre-tonic syllables, if any, which provides abrupt falling on a stressed syllable. The second accent is on the word form Islambek $\nearrow$ and is pronounced with a rising pitch on the final stressed syllable of the IC-3 type, marking the topic of the utterance. Next, the third accented word form health $\boldsymbol{C}$, is a member of the attributive group not dangerous to life and health $\nearrow$. It is pronounced with a rising pitch on the stressed syllable and a falling pitch on the post-tonic of the type IC-3 (Figure 1).

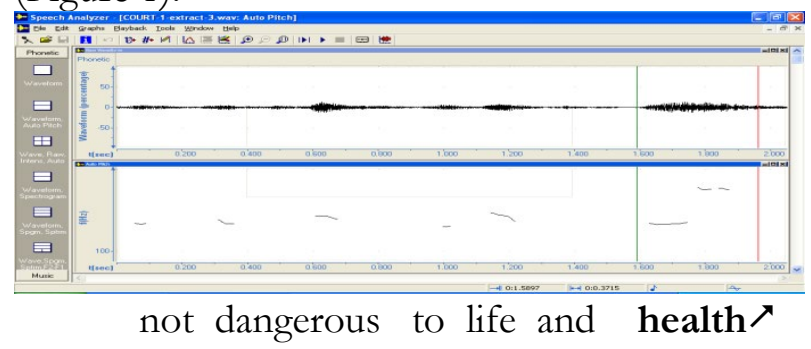

Figure 1. Intonation contour of the attributive group.

The ascending intonation IC-3 in this case is an ascending accent of incompleteness. That is, it does not carry a local function, nor a function 
relating to the formation of a separate speech act, but a discursive function. In other words, the rising intonation on this accent bearer does not mark one of the communicative components, such as topic, as in the word form Islambek $\nearrow$, but provides connectivity of discourse, it indicates that this fragment is not the last piece of text, and is followed by a sequel.

We note that the whole part preceding the accented word form, as is clearly seen in the graph, is spoken practically on one level tone, without sharp frequency fluctuations. The fourth accented word form government representative $\downarrow \nearrow$ is pronounced with a falling tone on the stressed syllable and a rising tone on the post-tonic syllables of the IC-4 type, being an accent of the incompleteness of the text. And the last, the fifth accent bearer in this sentence, the word form duties $\downarrow$, is pronounced with a smooth falling tone on the stressed syllable and the subsequent post-tonic syllables of the IC-1 type, marking the end of the sentence.

\section{Contrast as a modifier of communicative meaning and implicature of non-obvious sense}

While reading the narrative of the committed crime, the state prosecutor repeated again the previously uttered attributive group:

(2) violence, not dangerous $\searrow$ to life and health $>$,

but placed the communicatively relevant accents differently: with a distinct accent on the post-positive adjective not dangerous, with a rising tone on its pre-tonic syllables and a falling tone on the stressed and post-stressed syllables of the IC-2 type (Figure 2 between the cursors).

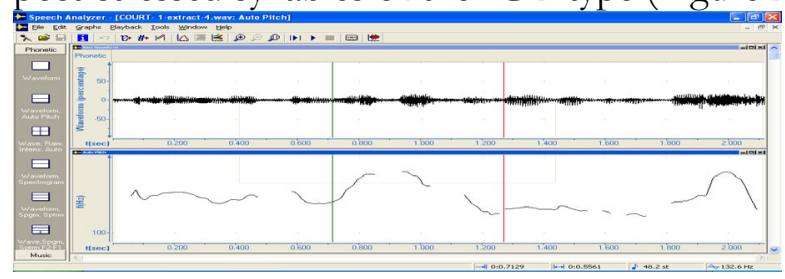

not dangerous $\searrow$ to life and health ${ }^{\nearrow}$

Figure 2. Rising and falling tone on the adjective.

In the last variant the new accent modifies the meaning of the communicative focus component and gains a new meaning - contrast. The semantics of contrast is related to a mental procedure of selecting from a variety of options associated with a component chosen by intonation and known to interlocutors (Yanko 2001:47). In our case this set may be limited, for example, to the options: not dangerous vs. 
dangerous. The prosodic expression of a contrastive focus with the contrast on an adjective, as is shown in the graphics of the right bottom panel of Figure 3, differs significantly from the non-contrastive version of the left bottom panel.

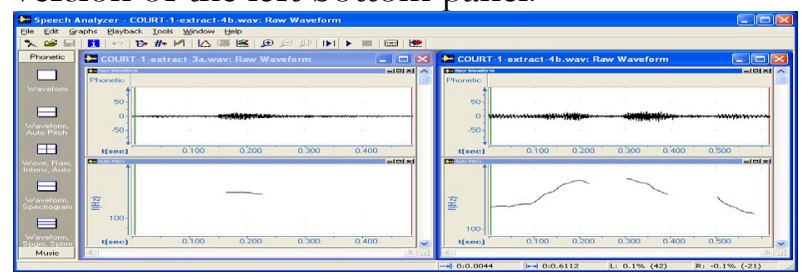

not dangerous not dangerous $\searrow$

Figure 3. Intonation contours of the non-contrastive and contrastive versions.

Measurements of intensity (an acoustic correlate of the volume) of both variants confirm highlighting of one of the utterances components. Thus, the intensity on the non-contrastive component is equal to $-19,4$ decibels $(\mathrm{dB})$, on the contrastive one is $-17 \mathrm{~dB}$, so the contrastive option sounds louder.

\section{Conclusion}

Thus, in the second example, physical measurements confirm the appearance of a new accent. Thereby there is the strategically intelligent singling out of the communicatively significant component of the sentence with the purpose, as stated above, of indicating by means of intonation the choice of that component. This choice has been made from the set of the possible variants associated with the selected component. (In our case it is two opposed variants: not dangerous vs. dangerous.) Meanwhile, in the use of this communicative strategy there is also another, probably more essential aspect: the prosecutor implicitly assesses, implicitly evaluates the committed crime according to the current legislation. The last hypothesis primarily may be confirmed by comparison with the current Penal Code, from which the above mentioned attributive construction is quoted and according to which the law provides for appropriate sanctions.

\section{References}

Austin, J. 1962. How to Do Things with Words. Oxford: Oxford University Press. Bryzgunova, E. 1980. Intonation. Russian grammar 1, 96-122.

Searle, J. 1976. A classification of illocutionary acts. Language in Society 5, 1-23.

Yanko, T. 2001. Russian Speech Communicative Strategies. Moscow: Slavic cultures languages. 